\title{
Obesity-associated stigma physiological markers of stress: evidence from the Dominican Republic
} and

\author{
Peter Muennig*,† and Kara Keating Bench \\ Department of Health Policy and Management, Columbia University, New York, NY, \\ USA
}

\begin{abstract}
Summary
Social stigma is increasingly recognized as a cause of stress-induced pathophysiology. We tested the hypothesis that stigma is associated with obesity-related morbidity, using a cohort of subjects from the Dominican Republic who value fat bodies over thin ones. We surveyed 87 subjects from Batey Algodon-a small region in the Dominican Republic where obesity is not stigmatized. We obtained information on ideal body norms, perceptions of one's own body and self-rated health. We also measured height, weight, waist circumference and blood pressure. We then performed linear regression analyses to ascertain the extent to which body mass index (BMI) and body norm perceptions were related to self-rated health and blood pressure. Self-rated health was strongly associated with one's satisfaction with his or her physical appearance $(p<0.001)$ and weight $(p$ $<0.001)$. As expected, self-rated health was not independently associated with BMI in this community, which does not stigmatize obesity. However, BMI was nevertheless associated with both systolic and diastolic blood pressure $(p<0.05)$. While de-stigmatizing obesity may improve perceptions of health, it might not significantly reduce the incidence of hypertension among heavier persons. Copyright (C) 2008 John Wiley \& Sons, Ltd.
\end{abstract}

\section{Key Words}

obesity; Stress; Social Desirability; Hypertension

\section{Introduction}

In the United States, obesity is heavily stigmatized, and there is evidence that this stigma affects human health (Cahnman, 1968; Chen \& Brown, 2005; Muennig, Jia, Lee, \& Lubetkin,

\footnotetext{
* Correspondence to: Peter Muenning, Columbria University, Department of Health Policy and Management, 600 West $_{168^{\text {th }}}$ Street, $6^{\text {th }}$ Floor, new York, NY 10032

†E-mail: pm124@columbia.edu
}

2008; Muennig, Lubetkin, Jia, \& Franks, 2006; Puhl \& Brownell, 2003). It has been hypothesized that stigma is stressful and that this stress is transduced in the brain into an autonomic response (McEwen, 1998; McEwen \& Mirsky, 2002; Muennig, Sohler, \& Mahato, 2007; Roy, 2004; Williams, 1999). Psychological stress, when chronic, can lead to autonomic dysregulation, predisposing afflicted persons to diabetes, heart disease and hypertension (McEwen, 1998; Roy, 2004). These are the very conditions for which obese people are at heightened risk. 
There is also evidence that obesity-associated stigma plays a large role in explaining the pathophysiology of obesity (Muennig et al., 2006, 2008). First, the difference between one's actual and ideal weight (a potential proxy measure of the extent to which that individual feels stigmatized) has been shown to be a stronger predictor of self-reported physical and mental health than body mass index (BMI) in the United States (Muennig et al., 2008). Moreover, groups who rate lower on body-image instruments and who report greater stigma associated with obesity (e.g. non-Hispanic whites and women) have much higher obesity-associated morbidity and mortality than groups who report lower levels of stigma or greater body satisfaction (e.g. African-Americans and men) (Cash, Phillips, Santos, \& Hrabosky, 2004; Fontaine, Redden, Wang, Westfall, \& Allison, 2003; Hebl \& Turchin, 2005; Muennig et al., 2006). Finally, obese persons have similar pro-thrombotic, proinflammatory serological profiles to chronically stressed individuals (McEwen, 1998; Muennig et al., 2007). These serological profiles may explain the higher incidence of hypertension, diabetes, stroke, heart disease and even cancer among obese persons, persons of African-American descent and those with low socio-economic status in the United States (McEwen, 1998; McEwen \& Mirsky, 2002; Muennig et al., 2007; Roy, 2004; Williams, 1999; Wong, Shapiro, Boscardin, \& Ettner, 2002).

If stigma explains a large portion of obesityassociated mortality, we would expect cultures with less obesity-associated stigma to have lower rates of obesity-associated illness. For instance, plumpness is so valued among women in Mauritania that many women are force-fed large quantities of food. Yet that country is among the countries with the lowest rates of diabetes (Ducorps, Baleynaud, Mayaudon, Castagne, \& Bauduceau, 1996).

Plumpness is also valued in Batey Algodón, a poor, rural region in the Dominican Republic. In this paper, we explore whether persons in Batey Algodón are susceptible to obesity-associated pathophysiology. We hypothesize: (1) subjects will self-report a preference for a heavier body; (2) BMI will not be associated with hypertension or self-rated health in this population (or the association will be weak); and (3) because bodyimage dissatisfaction for one reason or another is endemic worldwide, general measures of body satisfaction will independently predict self- rated health and hypertension. If all three hypotheses are correct, it would be reasonable to conclude that obesity-associated stigma is a major explanatory variable in the obesity-health association.

\section{Methods}

We surveyed 87 out of 93 adult residents between the ages of 25 and 64 of Batey Algodón in the Dominican Republic. The majority of residents are Haitian-Dominicans who come to the Dominican Republic for work harvesting sugar cane. All subjects were asked to sign informed consent. This study received approval from the Institutional Review Board at the Centro Nacional de Investigaciones en Salud Materno Infantil.

The survey employed the World Health Organization STEPWise approach to chronic disease risk factor surveillance (instrument v2.0 6) with questions added to ascertain body norms. The survey had three components: (1) a general health and sociodemographic profile; (2) questions pertaining to cultural body norms as well as perceptions of one's own body size; and (3) a medical examination component consisting of height, weight, waist circumference and three consecutive blood pressure readings. All surveys were administered by local health promoters trained in the STEPS methodology. The general demographics of the cohort by body size preference are described in Table I.

Regression models utilized self-rated health, diastolic blood pressure (mean of three measurements) and systolic blood pressure (mean of three measurements) as continuous dependent variables. Covariates considered for inclusion were age (25-64 years), gender (male or female), educational attainment (0-22 years), smoking (yes/ no), recreational exercise (yes/no), diet (measured as number of days per week fruit is consumed and number of days per week vegetables are consumed), alcohol consumption (1-2 glasses, abstains, <2 glasses), BMI ( $\left.\mathrm{m} / \mathrm{kg}^{2}\right)$, waist circumference $(\mathrm{cm})$, subjects' perception of their own physical appearance $(1=$ most content, $10=$ least content), perception of their own weight $(1=$ most content, $10=$ least content) and perception of body mass in general ( $1=$ fat is best, $10=$ thin is best). However, specification and sample size were improved when alcohol, diet and exercise were dropped in most models. Thus, final models 


\section{Obesity-associated stigma and physiological markers of stress}

Table I. Basic demographic characteristics of the sample by body type preference (rated on a scale of 1 to 10 with $1=$ 'fat is best' and $10=$ 'thin is best').

\begin{tabular}{|c|c|c|c|}
\hline & Prefers fat body & Prefers average body & Prefers thin body \\
\hline Number (row \%) & $38(45 \%)$ & $29(34 \%)$ & $18(21 \%)$ \\
\hline Mean age & 40 & 48 & 44 \\
\hline$\%$ Female & 55 & 59 & 61 \\
\hline Years of education completed & 5.2 & 4.0 & 5.1 \\
\hline Vegetable consumption* & 3.2 & 2.6 & 2.7 \\
\hline$\%$ Smoke & 21 & 28 & 17 \\
\hline$\%$ Exercise & 24 & 21 & 28 \\
\hline$\%$ Drink & 45 & 34 & 39 \\
\hline \multicolumn{4}{|l|}{ Mean blood pressure } \\
\hline Systolic & 119 & 126 & 114 \\
\hline Diastolic & 72 & 72 & 69 \\
\hline Body mass index & 27 & 26 & 25 \\
\hline Waist circumference in centimetres & 86 & 86 & 81 \\
\hline$\%$ Excellent or good self-rated health & 35 & 21 & 35 \\
\hline Perceive own weight ${ }^{\dagger}$ & 89 & 76 & 78 \\
\hline Perceive own physical appearance ${ }^{\dagger}$ & 84 & 55 & 78 \\
\hline
\end{tabular}

" Number of days per week vegetables are consumed.

${ }^{\dagger}$ Percentage of subjects within column category responding in the top $30 \%$ on a 10 -point 'most content' to 'least content' scale.

included age, sex, smoking and educational attainment, alongside the variables of interest (e.g. BMI, perception of their own weight). There were more missing variables for waist circumference than for BMI, so waist circumference was used only as a covariate in secondary analyses.

Finally, there were 16 (18\% of the sample) missing age values, and the majority of the sample did not know the year in which they were born. We conducted secondary analyses using imputed age values, but the results were not substantively changed. We therefore conducted all analyses with age as a missing value. Nevertheless, missing age values reduced the sample size to 66 subjects for most analyses.

\section{Results}

Table I presents the basic socio-demographic characteristics of the cohort by body type preference category. Column values represent responses to a $1-10$ scale ranking perception of body mass in general ( $1=$ 'fat is best' and $10=$ 'thin is best'). While none of the between-group differences were statistically significant, those who feel fat bodies are superior to thin bodies tend to be younger, male, heavier (both in terms of BMI and waistline), eat more vegetables on a weekly basis, drink and feel better about their own weight and physical appearance. They also exercise less.

Table II describes the relationship between the outcome variables of interest (self-rated health, systolic blood pressure and diastolic blood pressure) and subjects' BMI, perceptions of their own weight and perceptions of their physical appearance. The extent to which subjects reported being content with their weight or their physical appearance in general was strongly predictive of selfrated health $(p<0.001)$. However, these measures were not significant predictors of either systolic or diastolic blood pressure. Controlling for body type preference did not substantively change the relationship between BMI and blood pressure. Nor did it substantively change the relationship between self-rated health and body image rating measures. On the other hand, BMI predicted both systolic $(p=0.01)$ and diastolic blood pressure $(p$ $=0.01$ ). There was no relationship between BMI and self-rated health.

In secondary analyses using waist circumference rather than BMI, waist circumference did not predict systolic blood pressure but did predict diastolic blood pressure. An analysis of outliers revealed that the relationship between BMI and systolic blood pressure was heavily influenced by a single outlier. This subject had multiple risk factors for hypertension including 2 


\section{P. Muennig and K. K. Bench}

Table II. Predictors of self-rated health, systolic blood pressure and diastolic blood pressure in the cohort of 87 subjects from the Batey Algodón, Dominican Republic.

\begin{tabular}{|c|c|c|c|}
\hline Dependent/covariate & $\beta$ & $p$ & $R^{2}$ \\
\hline \multicolumn{4}{|l|}{ Self-rated health* } \\
\hline Body mass index & 0 & 0.8 & 0.02 \\
\hline Perception of own weight ${ }^{\dagger}$ & 0.22 & $>0.001$ & 0.27 \\
\hline Perception of physical appearance ${ }^{\dagger}$ & 0.18 & 0.004 & 0.15 \\
\hline \multicolumn{4}{|l|}{ Systolic blood pressure } \\
\hline Body mass index & 0.75 & 0.01 & 0.24 \\
\hline Perception of own weight ${ }^{\dagger}$ & -1.42 & 0.18 & 0.18 \\
\hline Perception of physical appearance ${ }^{\dagger}$ & 0.62 & 0.57 & 0.16 \\
\hline \multicolumn{4}{|l|}{ Diastolic blood pressure } \\
\hline Body mass index & 0.77 & 0.01 & 0.17 \\
\hline Perception of own weight ${ }^{\dagger}$ & 0.48 & 0.67 & 0.07 \\
\hline Perception of physical appearance ${ }^{\dagger}$ & 1.31 & 0.24 & 0.09 \\
\hline
\end{tabular}

All models include age, gender, smoking and education as well as the variable in the first column (e.g. body mass index).

* On a scale of 1 to 4 , where $1=$ excellent and $4=$ poor.

${ }^{\dagger}$ On a scale of 1 to 10 , where $1=$ most content and $10=$ least content.

years of education and a diet that included no fruits or vegetables. Removing this subject reduced the association to marginally significant $(p<0.1)$.

\section{Discussion}

In the United States, obese persons report extremely high levels of stigmatization and discrimination. For instance, among one group of formerly obese persons asked to choose between blindness or obesity, $89 \%$ chose blindness (Rand \& Macgregor, 1991). Discrimination is also pervasive; there is evidence that parents discriminate against their obese children, doctors against their obese patients and husbands against their obese wives (Chen \& Brown, 2005; Puhl \& Brownell, 1991). Given the potential relationship between stigma/discrimination and physical or mental health (Link \& Phelan, 2001, 2006; Williams, 1999), it is logical that obesity-associated stigma plays a role in the pathophysiology of obesity. Indeed, one recent study demonstrated that a measure of body dissatisfaction predicted measures of self-reported physical and mental health independent of BMI (Muennig et al., 2008).

If stigmatization of heavy bodies is a determinant of obesity-associated health problems, we would expect to find little or no association between obesity and measures of health in a society that does not stigmatize obesity. We nonetheless found that BMI remained a predictor of both systolic and diastolic hypertension within a community that tends to value heavier body types. As might be expected in a population that does not stigmatize obesity, we did find that the relationship between BMI and measures of hypertension was not modified by subjects' self-reported preference for fat versus thin body types.

The obesity-associated stigma model suggests that obesity-associated stigma and negative bodyimage work synergistically to disrupt autonomic processes (Muennig, 2008). We found evidence that satisfaction with one's weight is an independent predictor of self-rated health. Likewise, discontent with one's physical appearance in general was a strong predictor of self-rated health. However, within our small sample, weight and body image satisfaction do not predict systolic or diastolic blood pressure.

In summary, we find evidence of a role of body image in predicting health but cannot conclude that it is a predictor of hypertension among overweight and obese persons. The strength of this study was that it contained data on subject sociodemograhic characteristics, health risk factors, body-image preferences and medical exam data. However, it was limited by a lack of breadth in the health outcomes collected, a small sample size and failure to collect data on subjects' skin colour, which could have confounded the 


\section{Obesity-associated stigma and physiological markers of stress}

observed relationship between BMI and blood pressure.

The lack of more extensive health outcomes (e.g. hemoglobin A1c) limited the conclusions that could be drawn about the relationship between obesity-associated health conditions (e. g. diabetes) and obesity in this particular sample. While obesity has been associated with diabetes, heart disease, hypertension, stroke and certain types of cancer, we were only able to estimate a global measure of morbidity (self-rated health) and two specific obesity-associated measures (systolic and diastolic blood pressure).

Another limitation is that, while self-rated health has been validated as a predictor of a wide range of health problems in the United States and Europe, including Latinos within the United States (Franks, Gold, \& Fiscella, 2003), it has not been validated for use in this specific subpopulation in the Dominican Republic. Moreover, it is conceivable that the relationship between subjects' body image and self-rated health is confounded by self-esteem. Subjects with low self-esteem may see their health as poorer than it actually is, and this bias may explain the observed association. We attempted to minimize this potential form of confounding by asking about subjects' self-rated health before asking their body-image ratings.

Sample size also proved to be a limitation in this study. The study was adequately powered to detect a minimally clinically significant difference in blood pressure (roughly $10 \mathrm{~mm} \mathrm{Hg}$ in both measures). However, the results of the relationship between BMI and systolic hypertension were largely dependent on a single subject with a blood pressure of 180/80. Removing this subject reduced the association to marginally significant $(p<$ $0.1)$.

Finally, it is conceivable that heavier subjects also had darker skin. If so, by measuring an association between BMI and blood pressure, we could have inadvertently measured an association between BMI and race-associated stigma. Raceassociated stigma is thought to be associated with hypertension (Franks, Muennig, Lubetkin, \& Jia, 2006; Jackson et al., 1996; Lillie-Blanton, Parsons, Gayle, \& Dievler, 1996; Turner \& Avison, 2003; Williams, 1999). However, this confounding may have been partially captured when controlling for educational attainment; many darker skinned subjects were likely to have been denied access to education (e.g. because of their Haitian origins or simple racism). Holding education constant greatly strengthened the association between BMI and blood pressure.

To our knowledge, this is the first study to examine the relationship between obesity and health using a sample in a community that does not stigmatize obesity. Our paper suggests that obesity-associated stigma plays a role as a determinant of self-rated health. However, we also find that stigma may not be a major explanatory variable in the BMI-hypertension association seen in the United States. This latter conclusion is drawn from (1) an observed association between BMI and hypertension in a population of persons for whom obesity does not appear to be heavily stigmatized and (2) an observed positive association between body image and self-rated health. Given the small sample size and the potential for sources of external confounding (which were unavoidable given the limited resources available for conducting this study), our results should be considered preliminary.

Finally, many researchers have called attention to the potentially problematic nature of diet and exercise campaigns, sometimes launched by public health officials, that emphasize thinness rather than health and fitness (Campos, Saguy, Ernsberger, Oliver, \& Gaesser, 2006). Our study at a minimum adds to the debate, showing a strong relationship between self-rated health and basic measures of body image. Thus, regardless of whether obesity-related stigma is central or peripheral in the BMI-health association, messages that degrade body image appear to have health effects.

\section{References}

Cahnman, W.J. (1968). The stigma of obesity. The Sociological Quarterly, 9(3), 283-299.

Campos, P., Saguy, A., Ernsberger, P., Oliver, E., \& Gaesser, G. (2006). The epidemiology of overweight and obesity: Public health crisis or moral panic? International Journal of Epidemiology, 35(1), 55-60.

Cash, T.F., Phillips, K.A., Santos, M.T., \& Hrabosky, J.I. (2004). Measuring 'negative body image': Validation of the Body Image Disturbance Questionnaire in a nonclinical population. Body Image, 1(4), 363-372.

Chen, E.Y., \& Brown, M. (2005). Obesity stigma in sexual relationships. Obesity Research, 13(8), 1393-1397.

Ducorps, M., Baleynaud, S., Mayaudon, H., Castagne, C., \& Bauduceau, B. (1996). A prevalence survey of diabetes in Mauritania. Diabetes Care, 19(7), 761-763.

Fontaine, K.R., Redden, D.T., Wang, C., Westfall, A.O., \& Allison, D.B. (2003). Years of life lost due to obesity. The Journal of the American Medical Association, 289(2), 187193. 


\section{P. Muennig and K. K. Bench}

Franks, P., Gold, M.R., \& Fiscella, K. (2003). Sociodemographics, self-rated health, and mortality in the US. Social Science \& Medicine, 56(12), 2505-2514.

Franks, P., Muennig, P., Lubetkin, E., \& Jia, H. (2006). The burden of disease associated with being African-American in the United States and the contribution of socio-economic status. Social Science \& Medicine, 62(10), 2469-2478.

Hebl, M.R., \& Turchin, J.M. (2005). The stigma of obesity: What about men. Basic Applied Social Psychology, 27(3), $267-275$.

Jackson, J.S., Brown, T.N., Williams, D.R., Torres, M., Sellers, S.L., \& Brown, K. (1996). Racism and the physical and mental health status of African Americans: A thirteen year national panel study. Ethnicity \& Disease, 6(1-2), 132-147.

Lillie-Blanton, M., Parsons, P.E., Gayle, H., \& Dievler, A. (1996). Racial differences in health: Not just black and white, but shades of gray. Annual Review of Public Health, 17, 411-448.

Link, B.G., \& Phelan, J.C. (2001). Conceptualizing stigma. Annual Review of Sociology, 27(1), 363-385.

Link, B.G., \& Phelan, J.C. (2006). Stigma and its public health implications. Lancet, 367(9509), 528-529.

McEwen, B.S. (1998). Protective and damaging effects of stress mediators. The New England Journal of Medicine, 338(3), 171-179.

McEwen, B.S., \& Mirsky, A.E. (2002). How socioeconomic status may 'get under the skin' and affect the heart. European Heart Journal, 23(22), 1727-1728.

Muennig, P. (2008). The body politic: The association between stigma and obesity-associated disease. BMC Public Health, 8, 128-141.

Muennig, P., Jia, H., Lee, R., \& Lubetkin, E. (2008). I think therefore I am: Perceived ideal weight as a determinant of health. American Journal of Public Health, 98(3), 501506.

Muennig, P., Lubetkin, E., Jia, H., \& Franks, P. (2006). Gender and the burden of disease attributable to obesity. American Journal of Public Health, 96(9), 16621668.

Muennig, P., Sohler, N., \& Mahato, B. (2007). Socioeconomic status as an independent predictor of physiological biomarkers of cardiovascular disease: Evidence from NHANES. Preventive Medicine, 45(1), 35-40.

Puhl, R., \& Brownell, K.D. (1991). Bias, discrimination, and obesity. Obesity Research, 9(12), 788-805.

Puhl, R.M., \& Brownell, K.D. (2003). Psychosocial origins of obesity stigma: Toward changing a powerful and pervasive bias. Obesity Reviews, 4(4), 213-227.

Rand, C.S., \& Macgregor, A.M. (1991). Successful weight loss following obesity surgery and the perceived liability of morbid obesity. International Journal of Obesity, 15(9), 577-579.

Roy, J.P. (2004). Socioeconomic status and health: A neurobiological perspective. Medical Hypotheses, 62(2), 222227.

Turner, R.J., \& Avison, W.R. (2003). Status variations in stress exposure: Implications for the interpretation of research on race, socioeconomic status, and gender. Journal of Health and Social Behavior, 44(4), 488-505.

Williams, D.R. (1999). Race, socioeconomic status, and health. The added effects of racism and discrimination. Annals of the New York Academy of Sciences, 896, 173188.

Wong, M.D., Shapiro, M.F., Boscardin, W.J., \& Ettner, S.L. (2002). Contribution of major diseases to disparities in mortality. The New England Journal of Medicine, 347(20), 1585-1592. 